\title{
Correction to: Association of the Careggi Collateral Score with 3-month modified Rankin Scale score after thrombectomy for stroke with occlusion of the middle cerebral artery
}

\author{
Manuel Cappellari ${ }^{1}$. $\cdot$ Valentina Saia ${ }^{2} \cdot$ Giovanni Pracucci $^{3}$ - Enrico Fainardi ${ }^{4}$. Arturo Consoli ${ }^{5,6}$. Sergio Nappini ${ }^{6}$. \\ Lucio Castellan $^{7} \cdot$ Sandra Bracco $^{8} \cdot$ Mauro Bergui $^{9} \cdot$ Mirco Cosottini $^{10} \cdot$ Alessandra Briatico Vangosa $^{11}$. \\ Sergio Vinci ${ }^{12}$. Maria Ruggiero ${ }^{13}$. Edoardo Puglielli ${ }^{14}$. Luigi Chiumarulo ${ }^{15}$. Giacomo Cester ${ }^{16}$. Chiara Comelli ${ }^{17}$. \\ Umberto Silvagni ${ }^{18}$. Daniele Morosetti ${ }^{19}$. Valentina Caldiera ${ }^{20} \cdot$ Nicola Cavasin $^{21} \cdot$ Valeria Ledda $^{22}$. \\ Giuseppina Sanfilippo ${ }^{23} \cdot$ Andrea Saletti $^{24}$ - Pietro Filauri ${ }^{25} \cdot$ Ivan Gallesio ${ }^{26} \cdot$ Nunzio Paolo Nuzzi $^{27}$. \\ Pitero Amistá ${ }^{28}$. Cecilia Zivelonghi ${ }^{1}$ - Mauro Plebani ${ }^{29} \cdot$ Marco Pavia $^{30}$. Daniele Romano ${ }^{31}$ - Francesco Biraschi ${ }^{32}$. \\ Roberto Menozzi ${ }^{33}$. Roberto Gasparotti ${ }^{34}$ - Andrea Giorgianni ${ }^{35}$. Andrea Zini ${ }^{36}$. Domenico Inzitari ${ }^{3}$. Danilo Toni ${ }^{37}$. \\ Salvatore Mangiafico ${ }^{6}$ on behalf of The IRETAS Group
}

Published online: 24 December 2021

(c) The Author(s), under exclusive licence to Springer-Verlag GmbH Germany 2021

\section{Correction to: Journal of Neurology} https://doi.org/10.1007/s00415-021-10898-8

The original version of this article unfortunately contained a mistake. The given name and family names of Enrico Fainardi were incorrectly structured.

The original article can be found online at https://doi.org/10.1007/ s00415-021-10898-8.

Manuel Cappellari

manuel_cappellari@libero.it

1 Stroke Unit, DAI di Neuroscienze, Azienda Ospedaliera Universitaria Integrata, Piazzale A. Stefani 1, 37126 Verona, Italy

2 Neurology and Stroke Unit, S. Corona Hospital, Pietra Ligure, Italy

3 Department of NEUROFARBA, Neuroscience Section, University of Florence, Florence, Italy

4 Dipartimento di Scienze Biomediche, Sperimentali e Cliniche, Neuroradiologia, Università degli Studi di Firenze, Ospedale Universitario Careggi, Firenze, Italy

5 Service de Neuroradiologie Diagnostique et Thérapeutique Hôpital Foch, Suresnes, France

6 Interventional Neurovascular Unit, Careggi University Hospital, Florence, Italy

7 Department of Neuroradiology, IRCCS Ospedale Policlinico San Martino, Genoa, Italy

8 Unit of Neuroimaging and Neurointervention, University Hospital of Siena, Siena, Italy
Enrico is given name and Fainardi is family name.

9 Interventional Neuroradiology Unit, Città della Salute e della Scienza-Molinette, Turin, Italy

10 Department of Translational Research and New Technologies in Medicine and Surgery, University of Pisa, Pisa, Italy

11 Diagnostic and Interventional Neuroradiology Unit-"SS. Annunziata" Hospital, Taranto, Italy

12 UOC Neuroradiology, Department of Biomedical Sciences and of Morphologic and Functional Images, University of Messina, Messina, Italy

13 Department of Neuroradiology, AUSL Romagna, M. Bufalini Hospital, Cesena, Italy

14 Vascular and Interventional Radiology Unit, Ospedale Civile Mazzini, Teramo, Italy

15 UOS Neuroradiologia Interventistica, AOU Consorziale Policlinico, Bari, Italy

16 Department of Diagnostic Imaging and Interventional Radiology, Neuroradiology, Padua University Hospital, Padua, Italy

17 Interventional Neuroradiology Unit, San Giovanni Bosco Hospital, Turin, Italy 
18 Interventional Neuroradiology Unit, Azienda Ospedaliera Annunziata, Cosenza, Italy

19 Department of Diagnostic Imaging and Interventional Radiology, University of Rome Tor Vergata, Rome, Italy

20 Department of Neuroradiology, S. Corona Hospital, Pietra Ligure, Italy

21 Neuroradiology Unit, Ospedale dell'Angelo, USSL3 Serenissima, Mestre, Italy

22 Vascular and Interventional Neuroradiology Department, Azienda Ospedaliera G. Brotzu, Cagliari, Italy

23 Radiologia e Neuroradiologia Diagnostica e Interventistica, IRCCS Policlinico San Matteo, Pavia, Italy

24 Interventional Neuroradiology Unit, University Hospital Arcispedale S. Anna, Ferrara, Italy

25 Neuroradiology Unit, Presidio Ospedaliero SS. Filippo e Nicola, Avezzano, Italy

26 Neuroradiological Unit, Department of Radiology, Azienda Ospedaliera "SS Antonio e Biagio e C. Arrigo", Alessandria, Italy

27 Humanitas Clinical and Research Center-IRCCS, Rozzano, MI, Italy
28 Neuroradiology Unit, Ospedale S. Maria Misericordia, Rovigo, Italy

29 Neuroradiology Unit, Azienda Ospedaliera Universitaria Integrata, Verona, Italy

30 Neuroradiology Unit, Fondazione Poliambulanza, Brescia, Italy

31 UOC Neuroradiologia, AUO S. Giovanni di Dio e Ruggi D’Aragona, Salerno, Italy

32 Department of Human Neurosciences, Interventional Neuroradiology, Università degli Studi di Roma Sapienza, Rome, Italy

33 Neuroradiology Unit, Ospedale Universitario, Parma, Italy

34 Neuroradiology Unit, Spedali Civili, Brescia, Italy

35 Neuroradiology Department, Ospedale di Circolo-ASST Sette Laghi, Varese, Italy

36 Department of Neurology and Stroke Center, IRCCS Istituto delle Scienze Neurologiche di Bologna, Maggiore Hospital, Bologna, Italy

37 Emergency Department Stroke Unit, Department of Human Neurosciences, Sapienza University of Rome, Rome, Italy 